\title{
PREVALENCE OF DYSLIPIDEMIA IN YOUNG ADULTS.
}

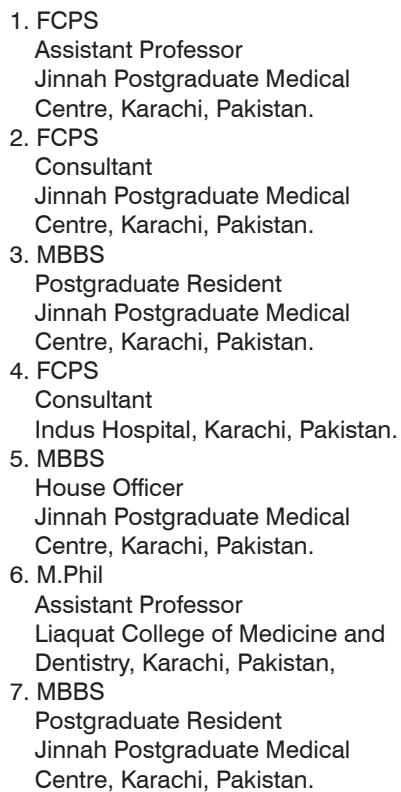

Correspondence Address:

Dr. Uzair Yaqoob

Lakhani Heights, Parsi Colony, Karachi.

ozair_91393@hotmail.com

Article received on:

10/08/2019

Accepted for publication:

$11 / 11 / 2019$
Mir Tahir Hussain Talpur ${ }^{1}$, Muhammad Tauqeer Katbar², Khalil Ullah Shabir ${ }^{3}$, Kashif Ullah Shabir ${ }^{4}$, Uzair Yaqoob ${ }^{5}$, Shah Jabeen ${ }^{6}$, Danish Zia ${ }^{7}$

ABSRACT... Objectives: This study will help us to understand what lipid profile is normally present in our population, if abnormal, timely screening, education, and proper management can be done. Study Design: Cross-sectional study. Setting: Jinnah Postgraduate Medical Centre (JPMC), Karachi, Pakistan. Period: November 2018-April 2019. Material \& Methods: For the lipid profile, intravenous blood samples were collected in testing bottles after overnight fasting of 9-12 hours and sent to the institutional laboratory for testing. Dyslipidemia was defined as abnormal levels of any of the following components of the lipid profile. Total cholesterol of $<200 \mathrm{mg} / \mathrm{dL}$ was considered "desirable" while that of $>240 \mathrm{mg} / \mathrm{dL}$ was termed as hypercholesterolemia. High-density lipoprotein (HDL) was considered as "low" when it was $<40$ mg/dL while Low-density lipoprotein (LDL) was considered as "optimal" when it was less than $100 \mathrm{mg} / \mathrm{dL}$. Triglycerides level $>150 \mathrm{mg} / \mathrm{dL}$ was identified as hypertriglyceridemia. We defined isolated dyslipidemias as abnormal levels of any of these. This study was assessed and approved by the institutional review board of JPMC. Results: 112 participants of age $<40$ and $>20$ years were registered with 62 males and 50 females. Prevalence of dyslipidemia was found to be $75.9 \%$ with a significantly high percentage in females $(p<0.05) .55 .4 \%$ of the participants had desirable serum cholesterol and $42.9 \%$ had hypertriglyceridemia. $58 \%$ of these had HDL levels $<40 \mathrm{mg} / \mathrm{dL}$ while $18.8 \%$ had high LDL levels. The mean serum cholesterol was significantly different in males and females $(p=0.018) .7 .1 \%$ of the total had isolated hypertriglyceridemia, $18.8 \%$ had isolated low HDL levels, and $46.4 \%$ had mixed hyperlipidemia, with significant differences between males and females in the former two. Conclusion: Dyslipidemia is a major risk factor for numerous systemic disorders and should be detected early in life for effective management before the appearance of symptoms. This study gave a high prevalence of dyslipidemia in asymptomatic young adults, with females being more frequently affected than females.

Key words: $\quad$ Dyslipidemia, Hyperlipidemia, LDL, HDL, Triglyceride, Young Adults.

Article Citation: Talpur MTH, Katbar MT, Shabir K, Shabir K, Yaqoob U, Jabeen S, Zia D. Prevalence of dyslipidemia in young adults. Professional Med J 2020; 27(5):987-993. DOI: 10.29309/TPMJ/2020.27.05.4040

\section{INTRODUCTION}

The term dyslipidemia signifies abnormal lipid or fat content in the blood, including triglycerides, phospholipids, cholesterol, lipoprotein, and other fats. Lipoproteinemia denotes fatty substances that circulate in the blood attached with proteins and when they exceed the normal level it is called hyperlipoproteinemia. ${ }^{1}$ Similarly, when cholesterol or triglycerides are increased, the term given to this condition is Hypercholesterolemia and Hypertriglyceridemia, respectively. Nonoptimal levels of Low-density lipoprotein (LDL) and High-density lipoprotein (HDL) cholesterol in young adults are independently associated with coronary atherosclerosis two decades later. ${ }^{2}$ The causes of hyperlipidemia are multiple that includes genetic factors, as in certain cases of familial hyperlipidemia; besides, other factors like cigarette smoking, alcohol, hypothyroidism, chronic kidney disease, obesity, medications, and dietary influences may also alter plasma lipid level. Plasma lipid levels are linked with normal functioning of heart, brain, and kidneys when present in normal range and when they become high, health implications of atherosclerosis, heart disease, stroke, and pancreatitis may occur. ${ }^{3}$

One study done on Hispanics that compared 
the old and young people presenting with Acute myocardial infarction (AMI) and to whom, percutaneous coronary intervention was done, it was concluded that high triglyceride level was the reason behind acute myocardial infarction. ${ }^{4}$ Another study was done in young adults of Assam, to determine the serum Total cholesterol (TC) level in blood and association of that cholesterol level with body mass index (BMI), concluded that young adults, especially who are overweight should be advised routine cholesterol testing so that preventive measures can be adopted to avoid hypercholesterolemia and its complications in future life. ${ }^{5}$

In a study done in Chongqing, China, to estimate the frequency and risk factors of dyslipidemia, 5375 residents aged $\geq 18$ years were selected. They found $35.5 \%$ prevalence of dyslipidemia (34.4\% among men and $37.6 \%$ among women). Out of these 2009 participants, 44.2\% of the participants had isolated hypertriglyceridemia, $14.7 \%$ had isolated hypercholesterolemia, $13.2 \%$ had mixed hyperlipidemia, and $28.0 \%$ had isolated low HDL cholesterol. The age group in males having the most cases of dyslipidemia was of 30-39 years (48.2\%). In a nutshell, they advised conducting proper intervention programs for reducing risk factors and routine screening programs to check for blood lipid levels. ${ }^{6}$

Another study conducted in three states of India where young people (age greater than 20 years) were selected, $13.9 \%$ had raised cholesterol level, $72.3 \%$ had decreased HDL, $11.8 \%$ had raised LDL, $29.5 \%$ had hypertriglyceridemia, and $79 \%$ had abnormalities in one of the lipid parameters. This is a huge percentage that was observed during this study. The high prevalence rate of dyslipidemia in states of India called for urgent lifestyle intervention strategies so that preventive measures are taken at a larger scale. ${ }^{7}$

Nowadays, undiagnosed underlying dyslipidemia is the most common cause of mortality and morbidity in young adults and that's because of atherosclerotic related changes leading to stroke, myocardial infarction, hypertension, and other metabolic syndromes. ${ }^{8}$
Although several studies have been done worldwide, there is variability in those publications. Assuming the high lipid levels in our population similar to what we found in literature, the results of this study will help us to understand what lipid profile is normally present in our population, if abnormal, timely screening, education, and proper management can be done. Stress should be given in national health care policies to prevent in order to prevent the hazards of Stroke, Myocardial infarction, and Metabolic Syndrome because of dyslipidemia.

\section{MATERIAL \& METHODS}

This is a descriptive, cross-sectional study conducted in the outpatient department of medicine, Jinnah Postgraduate Medical Centre, Karachi, Pakistan, from November 2018 to April 2019. Both male and female young adults (defined in this study as those aged 21-39 years) after taking informed consent were included in the study. Pregnant women, patients with diagnosed liver diseases, familial hyperlipidemias, diabetes, hypertension, nephrotic syndrome, and those using any lipid-lowering agents like statins were excluded from the study. Those not willing to participate were also excluded.

112 patients were gathered in the assigned duration. Limited information was collected i.e. their age, gender, and the parameters of their fasting lipid profile including total cholesterol, triglycerides, HDL, and LDL cholesterol.

For the lipid profile, intravenous blood samples were collected in testing bottles (containing an anticoagulant agent) after overnight fasting of 9-12 hours and sent to the institutional laboratory for testing by an automated spectrophotometer and enzymatic colorimetric method according to the guidelines of National Committee for Clinical Laboratory Standards. ${ }^{9}$

Dyslipidemia was defined according to the National Cholesterol Education Project (NCEP) Adult Treatment Panel III as abnormal levels of any of the following components of the lipid profile. TC of less than $200 \mathrm{mg} / \mathrm{dL}$ was considered "desirable" while that of $>240 \mathrm{mg} / \mathrm{dL}$ was termed 
as hypercholesterolemia. HDL was considered as "low" when it was $<40 \mathrm{mg} / \mathrm{dL}$ while LDL was considered as "optimal" when it was less than $100 \mathrm{mg} / \mathrm{dL}$. Triglycerides level $>150 \mathrm{mg} / \mathrm{dL}$ was identified as hypertriglyceridemia. ${ }^{10}$ We defined isolated dyslipidemias as abnormal levels of any one of these factors. However, mixed dyslipidemia was the term given to abnormalities in more than one of these. Although borderline levels are alarming, we did not consider those in the classification of hyperlipidemias.

The statistical analysis was performed using SPSS version 23.0. Mean and standard deviations were recorded for age and the lipid profile parameters. The data were further categorized according to age group. Post-stratification, Chi-square and student t-test was applied. P-value $<0.05$ was considered significant.

\section{RESULTS}

In this short study population of 112 , we had 62 males (55.4\%) and 50 females (44.6\%). We only gathered participants of age less than 40 years and greater than 20 years with a mean age of $32.5 \pm 5.2$ years.

In this study, we found that $75.9 \%$ of young adults had dyslipidemia. We first distributed the population according to categories of lipid levels made by the NCEP Adult Treatment Panel III ${ }^{10}$, which placed $56 \%$ of the participants in the serum cholesterol range of $<200 \mathrm{mg} / \mathrm{dL}$ (desirable/ low) and $21.4 \%$ in that of $>239 \mathrm{mg} / \mathrm{dL}$ (high). When triglyceride levels were analyzed, $42.9 \%$ had it equal or above the critical level. $58 \%$ of participants assigned to the study had HDL levels $<40 \mathrm{mg} / \mathrm{dL}$ while four of them had it above $60 \mathrm{mg} /$ $\mathrm{dL}$. On the other hand, on grouping LDL levels we had $31.3 \%$ of population with LDL levels $<100$ $\mathrm{mg} / \mathrm{dL}, 50.0 \%$ in a range of $100-159 \mathrm{mg} / \mathrm{dL}$, and $18.8 \%$ above $>159 \mathrm{mg} / \mathrm{dL}$ (Figure-1).
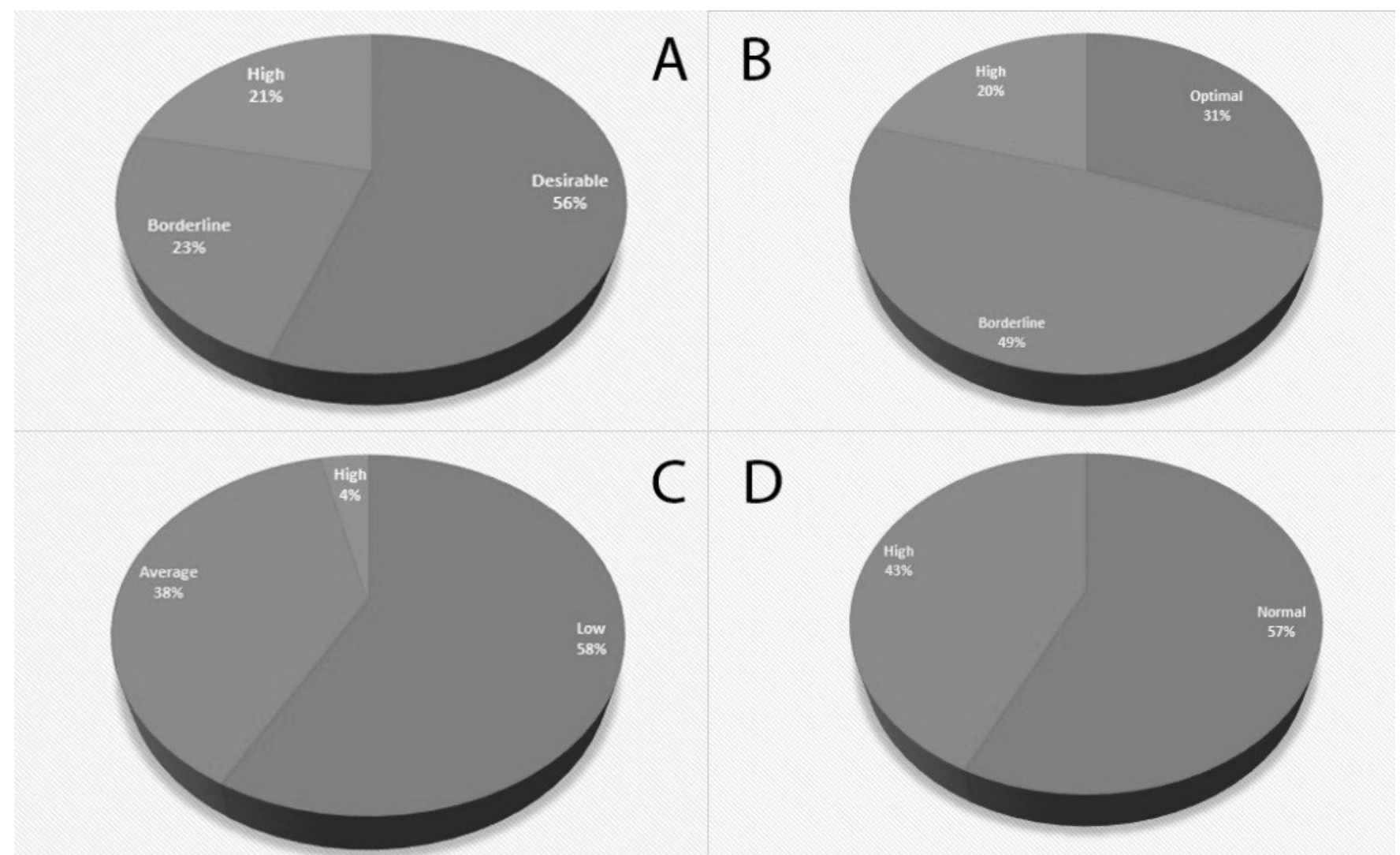

Figure-1. Pie charts showing percentage distribution of population according to the different sub-groups of plasma lipid levels of A: total cholesterol; B: low-density lipoprotein; C: high-density lipoprotein; D: triglycerides 


\begin{tabular}{|l|c|c|c|c|}
\hline \multicolumn{1}{|c|}{ Characteristics } & Total $(\mathbf{n = 1 1 2})$ & Male $(\mathbf{n}=\mathbf{6 2})$ & Female $(\mathbf{n}=\mathbf{5 0})$ & P-Value \\
\hline Total cholesterol mean + SD (mg/dL) & $209.17+99.96$ & $189.21+66.295$ & $233.92+126.635$ & 0.018 \\
\hline Triglycerides mean + SD (mg/dL) & $171.94+125.81$ & $169.90+114.642$ & $174.46+139.584$ & 0.850 \\
\hline $\begin{array}{l}\text { High-density lipoprotein mean + SD } \\
\text { (mg/dL) }\end{array}$ & $38.91+9.89$ & $38.98+9.720$ & $38.82+10.213$ & 0.934 \\
\hline $\begin{array}{l}\text { Low-density lipoprotein mean + SD } \\
(\mathrm{mg} / \mathrm{dL})\end{array}$ & $129.54+80.86$ & $125.13+55.293$ & $135.00+104.333$ & 0.522 \\
\hline Dyslipidemia, \% (95\% Cl) & $75.9(65.9,82.7)$ & $74.2(61.5,84.5)$ & $76.0(61.8,86.9)$ & 0.000 \\
\hline $\begin{array}{l}\text { Isolated hypertriglyceridemia, \% (95\% } \\
\text { Cl) }\end{array}$ & $7.1(3.1,13.6)$ & $8.1(2.7,17.8)$ & $6.0(1.3,16.5)$ & 0.000 \\
\hline Isolated low HDL, \% (95\% Cl) & $18.8(12.0,27.2)$ & $17.7(9.2,29.5)$ & $20.0(10.0,33.7)$ & 0.000 \\
\hline Mixed hyperlipidemia, \% (95\% Cl) & $46.4(37.0,56.1)$ & $45.2(32.5,58.3)$ & $48.0(33.7,62.6)$ & 0.508 \\
\hline \multicolumn{2}{|c|}{ Table-I. Mean serum lipid concentrations (in total and based on gender) and prevalence of dyslipidemia } \\
\hline
\end{tabular}

Table-I shows the descriptive statistics of lipid profile in total and the distribution according to male and female gender. The mean total cholesterol overall was $209.17 \pm 99.96$ with significant differences in males and females $(p=0.018$ ) while the differences in triglyceride levels, HDL and LDL between males and females were non-significant. In Table-I, the prevalence of dyslipidemia and the different types of hyperlipidemias is also shown with $75.9 \%$ overall, and a significantly high percentage of dyslipidemia in females ( $p<0.05$ ). $7.1 \%$ of the total had isolated hypertriglyceridemia, $18.8 \%$ had isolated low HDL levels, and $46.4 \%$ had mixed hyperlipidemia, with significant differences between males and females in the former two.

\section{DISCUSSION}

Dyslipidemia has become a major public health concern. Its prevalence is rising at a steady pace particularly in developing countries. ${ }^{11}$ The increasing prevalence of dyslipidemia is associated with several factors of which many are modifiable and are dependent on socioeconomic, cultural, and ethnic characteristics. ${ }^{6}$ Changes in lifestyle and diet contribute to a significant portion of risk factors associated with dyslipidemia. Risk factors for dyslipidemia are lack of physical activity, obesity particularly central obesity, metabolic syndrome, hypertension, old age, and a diet rich in saturated fats and cholesterol. ${ }^{12}$ Dyslipidemia can be effectively controlled by pharmacological intervention but more importantly through dietary and lifestyle modification. ${ }^{13,14}$ Unfortunately, lack of awareness and appropriate therapeutic intervention and management are emerging as barriers in preventing complications related to dyslipidemia.

Dyslipidemia is a major pathogenic risk factor for atherosclerotic cardiovascular disease (CVD). Various cardiovascular incidents reported are all associated with uncontrolled dyslipidemia. ${ }^{15,16}$ Surveys conducted in China showed that the incidence of ischemic CVD is significantly reduced by controlling lipid profile among individuals, therefore decreasing the mortality and morbidity associated with it ${ }^{17}$. The asymptomatic nature of dyslipidemia is also a concerning issue. Hence, early screening and preventive measures are equally important in controlling it. ${ }^{18,19}$

A National Health and Nutrition Examination Survey was conducted in the United States (U.S.) from 2003 through 2006 in which the proportion of the U.S. population with abnormalities in the lipid profile was assessed. In this study, it was stated by Toth et al. that about 53 percent of U.S. adults have at least one lipid abnormality. ${ }^{12} \mathrm{~A}$ similar survey that took place in China reported that approximately 41.9 percent of the Chinese population was found to have dyslipidemia. ${ }^{20}$ These studies reflected the threat dyslipidemia pose on the general health of the population. In our study, we have succeeded to show that approximately $75.9 \%$ of young adults had dyslipidemia which is significantly greater than the prevalence described in the above countries. 
The prevalence of dyslipidemia increases with age; however, it is essential to note that it also affects younger adults. ${ }^{21}$ Moreover, the process of atherosclerosis starts early in life. A compilation of various observations made by the Bogalusa Heart Study established an association between coronary atherosclerosis and cardiovascular risk factors in young people, among which serum LDL and serum triglyceride concentration were a significant factor along with many others ${ }^{22,23}$ Therefore, young adults with abnormal levels of lipids are at an increased risk of developing cardiovascular complications, such as coronary heart disease later in life. Considering the lack of literature on dyslipidemias in young adults and its importance in early detection of cardiovascular risks, we have highlighted the significance of identifying dyslipidemia in young adults.

Our study showed that the prevalence of dyslipidemia is higher in females as compared to males. Our result is coherent with a cross-sectional survey conducted in Chongqing, China. In this study, the prevalence of dyslipidemia in women was $37.6 \%$ as compared to $34.4 \%$ in men. This was primarily due to differences in the frequency of overweight, obesity, and central obesity. ${ }^{6}$ In a study conducted in Iran, the incidence of dyslipidemia was $37.4 \%$ for males and $55.4 \%$ for females and therefore, higher in females which is consistent with the result of our study. ${ }^{24,25}$

However, a vast literature provides evidence that the prevalence of dyslipidemia is much higher in men as compared to women. This was shown by a cross-sectional study by Pan et al. in which the incidence of dyslipidemia was higher in males than in females (41.92\% vs $32.47 \%) .{ }^{19}$ Likewise in 2002, the Chinese national nutrition and health survey concluded the prevalence of dyslipidemia in Chinese adults as $22.2 \%$ and $15.9 \%$ in males and females respectively. ${ }^{26}$ Moreover, a systemic review on dyslipidemia in Chinese adults published between 2003 and 2013 stated that dyslipidemia was more common in men than in women. ${ }^{27}$ Prior researches have well established that abnormal lipid profile is more prevalent in men as compared to women. ${ }^{28}$ This could be due to a higher frequency of cigarette smoking and more consumption of alcohol and high cholesterol food in men ${ }^{27}$

The prevalence of isolated hypercholesterolemia, isolated hypertriglyceridemia, and isolated low HDL cholesterol in the total population of our study was $0.9 \%, 7.1 \%$, and $18.8 \%$ respectively. In the study conducted by Pan et al. this prevalence was $2.9 \%, 11.9 \%$, and $7.4 \%$ respectively. ${ }^{19}$ Extensive surveys in China organized from 2002 to 2010 revealed that low HDL-C and hypertriglyceridemia were the two major types of dyslipidemia in Chinese adults. ${ }^{29,30}$ These findings were reinforced in a meta-analysis by Huang et al. ${ }^{27}$ However, these trends are different in western countries where high cholesterol and high LDL-C were more common forms of dyslipidemia. ${ }^{19}$ This was largely due to a high dietary fat and cholesterol intake in American residents.

Furthermore, according to our study isolated low HDL was higher in females as compared to males whereas isolated hypertriglyceridemia was higher in males than females. The results are consistent with the Korean National Health and Nutrition Survey in which the large difference in HDL-C across six countries was secondary to smoking, alcohol consumption, age, fat intake, BMI and educational level. Gender difference in HDL-C is partly explained by high levels of estrogen in women. This explains the lower mortality rate due to cardiovascular diseases in the female gender. Hence HDL-C is believed to be protective. ${ }^{31}$

The early detection of dyslipidemia is important for implementing management strategies. These strategies can in return reduce the risk of cardiovascular diseases that may manifest later in life. Certain lifestyle modifications and lipidlowering drugs can be effective on individuals with abnormal lipid profiles. However, literature regarding the benefits of early screening and initiation of treatment is sparse. A systematic review in the United States by Chou et al. stated that there was no prior study that could provide strong evidence for the effects of screening or treatment on clinical outcomes in younger adults. Moreover, the benefit of early treatment was not clear. Therefore trials conducted on a big scale 
are needed to find evidence for the benefits and harms of dyslipidemia screening or treatment in younger adults. ${ }^{21}$

There are some limitations to this study, first being that this is a cross-sectional study with no causal associations between any of the variables. Secondly, this survey is limited by a small sample size, and data collection in a single tertiary care setup. Another restriction was that the potential risk factors of dyslipidemia were not taken into consideration.

\section{CONCLUSION}

Dyslipidemia, being a major risk factor for many systemic diseases should be detected early in life so that effective management is carried out before the start of actual manifestations. This study gave a high prevalence of dyslipidemia in asymptomatic young adults, with females being more frequently affected than females.

\section{Copyright@ 11 Nov, 2019.}

\section{REFERENCES}

1. Ahmed SM, Clasen ME, Donnelly JE. Management of dyslipidemia in adults. Am Fam Physician. 1998 May; 57(9):2192-204, 2207-8.

2. Pletcher MJ, Bibbins-Domingo K, Liu K, Sidney S, Lin F, Vittinghoff E, et al. Nonoptimal lipids commonly present in young adults and coronary calcium later in life: the CARDIA (Coronary Artery Risk Development in Young Adults) study. Ann Intern Med. 2010 Aug; 153(3):137-46.

3. Segen J. Concise Dictionary of modern medicine. 2nd ed. Maidenhead, United States: McGraw-Hill Education; 2005.

4. Essilfie G, Shavelle DM, Tun H, Platt K, Kobayashi R, Mehra A, et al. Association of elevated triglycerides and acute myocardial infarction in young Hispanics. Cardiovasc Revasc Med. 2016 Dec; 17(8):510-4.

5. Nath S, Jahan W. A study of serum cholesterol level in young adults and its relation to body mass index and waist-circumference. Indian J Physiol Pharmacol. 2014; 58(2):152-6.
6. Qi L, Ding X, Tang W, Li Q, Mao D, Wang Y. Prevalence and risk factors associated with dyslipidemia in Chongqing, China. Int J Environ Res Public Health. 2015 Oct; 12(10):13455-65.

7. Joshi SR, Anjana RM, Deepa M, Pradeepa R, Bhansali $A$, Dhandania VK, et al. Prevalence of dyslipidemia in urban and rural India: The ICMR-INDIAB study. PLoS One. 2014; 9(5):e96808.

8. Srikanth S, Deedwania P. Management of dyslipidemia in patients with hypertension, diabetes, and metabolic syndrome. Curr Hypertens Rep. 2016 Oct; 18(10):76.

9. CLSI. Procedures for the handling and processing of blood specimens for common laboratory tests; Approved guideline - fourth edition. CLSI document H18-A4. Wayne, PA; 2010.

10. Grundy SM, Becker DM, Clark LT, Cooper RS, Denke MA, Howard J, et al. Detection, evaluation, and treatment of high blood cholesterol in adults (Adult Treatment Panel III). Circulation. 2002; 106:3143--3421.

11. Sun G-Z, Li Z, Guo L, Zhou Y, Yang H-M, Sun Y-X. High prevalence of dyslipidemia and associated risk factors among rural Chinese adults. Lipids Health Dis. $2014 ; 13: 189$.

12. Toth PP, Potter D, Ming EE. Prevalence of lipid abnormalities in the United States: The National Health and Nutrition Examination Survey 2003-2006. J Clin Lipidol. 2012; 6(4):325-30.

13. MRC/BHF Heart Protection Study of cholesterol lowering with simvastatin in 20,536 high-risk individuals: A randomised placebo-controlled trial. Lancet (London, England). 2002; 360(9326):7-22.

14. Grundy SM, Cleeman JI, Merz CNB, Brewer HBJ, Clark LT, Hunninghake DB, et al. Implications of recent clinical trials for the National Cholesterol Education Program Adult Treatment Panel III Guidelines. J Am Coll Cardiol. 2004; 44(3):720-32.

15. Penalva RA, Huoya M de O, Correia LCL, Feitosa GS, Ladeia AMT. Lipid profile and intensity of atherosclerosis disease in acute coronary syndrome. Arq Bras Cardiol. 2008; 90(1):24-30.

16. McDonald M, Hertz RP, Unger AN, Lustik MB. Prevalence, awareness, and management of hypertension, dyslipidemia, and diabetes among United States adults aged 65 and older. J Gerontol A Biol Sci Med Sci. 2009; 64(2):256-63.

17. [Chinese guidelines on prevention and treatment of dyslipidemia in adults]. Zhonghua Xin Xue Guan Bing Za Zhi. 2007; 35(5):390-419. 
18. He H, Yu Y, Li Y, Kou C, Li B, Tao Y, et al. Dyslipidemia awareness, treatment, control and influence factors among adults in the Jilin province in China: A crosssectional study. Lipids Health Dis. 2014; 13:122.

19. Pan L, Yang Z, Wu Y, Yin R-X, Liao Y, Wang J, et al. The prevalence, awareness, treatment and control of dyslipidemia among adults in China. Atherosclerosis. 2016; 248:2-9.

20. Zhao W, Zhang J, You Y, Man Q, Li H, Wang C, et al. [Epidemiologic characteristics of dyslipidemia in people aged 18 years and over in China]. Zhonghua Yu Fang Yi Xue Za Zhi. 2005; 39(5):306-10.

21. Chou R, Dana T, Blazina I, Daeges M, Bougatsos C, Jeanne TL. Screening for dyslipidemia in younger adults: A systematic review for the U.S. preventive services task force. Ann Intern Med. 2016; 165(8):5604.

22. Berenson GS, Srinivasan SR, Bao W, Newman WP 3rd, Tracy RE, Wattigney WA. Association between multiple cardiovascular risk factors and atherosclerosis in children and young adults. The Bogalusa Heart Study. N Engl J Med. 1998; 338(23):1650-6.

23. Loria CM, Liu K, Lewis CE, Hulley SB, Sidney S, Schreiner $\mathrm{PJ}$, et al. Early adult risk factor levels and subsequent coronary artery calcification: The CARDIA Study. J Am Coll Cardiol. 2007; 49(20):2013-20.

24. Sadeghi M, Talaei M, Oveisgharan S, RabieiK, Dianatkhah $M$, Bahonar $A$, et al. The cumulative incidence of conventional risk factors of cardiovascular disease and their population attributable risk in an Iranian population: The Isfahan Cohort Study. Adv Biomed Res. 2014; 3:242.
25. Latifi SM, Karandish M, Shahbazian HB, Chinipardaz R, Sabet A, Pirani N. A survey of the incidence of dyslipidemia and its components in people over 20 years old in Ahvaz: A cohort study 2009-2014. Diabetes Metab Syndr. 2017; 11 Suppl 2:S751-4.

26. Wu Y, Huxley R, Li L, Anna V, Xie G, Yao C, et al. Prevalence, awareness, treatment, and control of hypertension in China: Data from the China National Nutrition and Health Survey 2002. Circulation. 2008; 118(25):2679-86.

27. Huang Y, Gao L, Xie X, Tan SC. Epidemiology of dyslipidemia in Chinese adults: meta-analysis of prevalence, awareness, treatment, and control. Popul Health Metr. 2014; 12(1):28.

28. Liu X, Yu S, Mao Z, Li Y, Zhang H, Yang K, et al. Dyslipidemia prevalence, awareness, treatment, control, and risk factors in Chinese rural population: the Henan rural cohort study. Lipids Health Dis. 2018; $17(1): 119$.

29. Yang W, Xiao J, Yang Z, Ji L, Jia W, Weng J, et al. Serum lipids and lipoproteins in Chinese men and women. Circulation. 2012; 125(18):2212-21.

30. Li J, Wang L, Li Y, Bi Y, Jiang Y, Mi S, et al. [Epidemiologic characteristics of dyslipidemia in Chinese adults 2010]. Zhonghua Yu Fang Yi Xue Za Zhi. 2012; 46(5):414-8.

31. Kim HJ, Park HA, Cho YG, Kang JH, Kim KW, Kang JH, et al. Gender Difference in the Level of HDL Cholesterol in Korean Adults. Korean J Fam Med. 2011; 32(3):17381.

\begin{tabular}{|c|c|c|c|}
\hline \multicolumn{4}{|c|}{ AUTHORSHIP AND CONTRIBUTION DECLARATION } \\
\hline Sr. \# & Author(s) Full Name & Contribution to the paper & Author(s) Signature \\
\hline 1 & Mir Tahir Hussain Talpur & & \\
\hline 2 & M. Tauqeer Katbar & & \\
\hline 3 & Khalil Ullah Shabir & $\begin{array}{l}\text { All authors have contributed } \\
\text { equally from the idea to the }\end{array}$ & \\
\hline 4 & Kashif Ullah Shabir & $\begin{array}{l}\text { completion of study with a little } \\
\text { more work by some according }\end{array}$ & \\
\hline 5 & Uzair Yaqoob & $\begin{array}{c}\text { to which the sequence is } \\
\text { made. }\end{array}$ & \\
\hline 6 & Shah Jabeen & & \\
\hline 7 & Danish Zia & & \\
\hline
\end{tabular}

